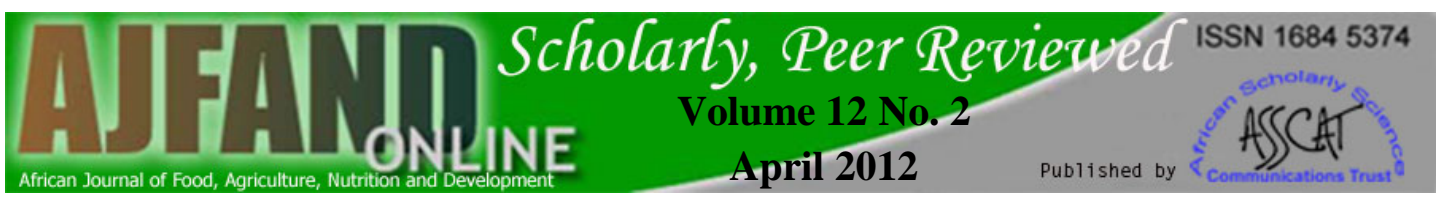

\title{
PROTEIN-ENERGY MALNUTRITION AND MALARIA ANTIBODY PROFILES IN PRE-SCHOOL CHILDREN IN WESTERN KENYA: A POTENTIAL DIAGNOSTIC TOOL
}

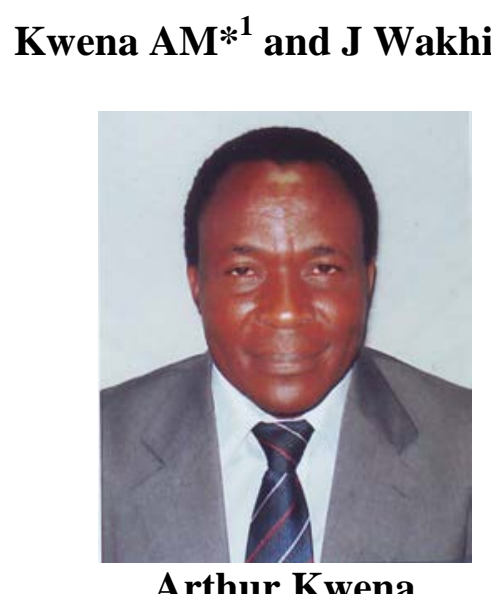

*Corresponding author: E-mail: arthurkwena@gmail.com

${ }^{1}$ Senior Lecturer, Department of Medical Biochemistry, School of Medicine, Moi University, P.O. Box 4606- 30100, Eldoret, Kenya.

${ }^{2}$ Associate Professor, Department of Medical Biochemistry, School of Medicine, Moi University, P.O. Box 4606- 30100, Eldoret, Kenya. 


\section{ABSTRACT}

Protein-energy malnutrition is a serious clinical condition with high prevalence in areas where Plasmodium falciparum is highly endemic such as western Kenya. There is a major need to determine the relationship between PEM and malaria antibody profiles especially in an area where malaria is endemic. The objective of this work, therefore, was to determine the association between PEM and specific malaria antibodies and the potential diagnostic value of the antibodies in children aged between 5 and 59 months. Cross- sectional surveys as well as analysis of sera for specific malaria antibodies were carried out at Asembo Division, Bondo District, Kisumu County, Nyanza Province. A total of sixty villages identified through random sampling with each household as the sampling unit were used for data collection. Two thousand, one hundred and twelve (2112) Children $<5$ years of age were sampled in three successive cross- sectional surveys: The first survey included children $<3$ years of age while the subsequent two surveys included children $<5$ years of age. Anthropometric measurements were carried out followed by finger prick blood sample for assay of antibodies in sera of the study children. Statistical variables (Odds Ratio, at 95\% CI) were determined using SPSS 11 and SAS computer packages. Both Multivariate and Bivariate analyses were carried out. Epi-info 2002 package was used to determine anthropometric variables. Demographic variables and malaria parasite counts were determined for all the children sampled. Circumsporozoite Surface Protein (CSP) IgG antibody was found to be significantly associated with stunting and underweight $(\mathrm{p}<0.05)$ but not with wasting. Liver Stage Antigen (LSA) IgG antibody was significantly associated with wasting only $(\mathrm{p}<0.05)$ while Merozoite Surface Protein (MSP) IgG antibody was not significantly associated with any malnutrition state. The mean concentration of CSP IgG was elevated in stunted, wasted and underweight in comparison to controls. Liverstage antigen 1 IgG was elevated in stunted children only as compared to controls, whereas MSP IgG was low in all PEM cases as compared to controls. Specific Plasmodium falciparum antibody profiles could accurately be used to determine the association between malaria and ProteinEnergy Malnutrition.

Key words: Malaria, Malnutrition, Children, Western Kenya 


\section{INTRODUCTION}

Protein-energy malnutrition (PEM) is a serious clinical condition with high prevalence in areas where malaria tropica (plasmodium falciparum) is highly endemic such as western Kenya [1]. It is associated with 40-60\% of deaths among under 5-year old children in developing countries [2]. Children with PEM and particularly those with oedematous malnutrition (Kwashiorkor) show an increased risk for a variety of infections [3]. Previous studies showed that plasmodium falciparum antibody profiles in children aged under six years was higher in well-nourished children than malnourished ones [3,4]. The interaction between PEM and Plasmodium falciparum malaria however is unclear as it is unknown whether the malaria antibodies namely Merozoite surface protein (MSP), Liverstage Antigen (LSA), and Circumsporozoite Surface Protein (CSP) can be used as a diagnostic tools. The antibodies could also shade some light on their potential as vaccines in Protein-energy malnourished children. Other studies have shown the relationship between malaria parasitaemia and PEM but none has specifically reported the link between PEM and malaria antibody profiles [5, 6].

\section{MATERIALS AND METHODS}

\section{Study area and population}

This study was conducted in parallel with a large study of the impact of permethrintreated bednets on childhood morbidity and mortality and provided the baseline information to determine the impact of this population-based intervention on anthropometric indices $[7,8]$. The bednet project was conducted in Asembo, Bondo District located on the shores of Lake Victoria, Western Kenya. The current nutritional study was conducted in 60 adjacent villages in an area of $130 \mathrm{~km}^{2}$. According to the bi-annual census conducted as part of the bednet project, approximately 5150 children aged $<5$ years live in this area at the time of the study. The population is ethnically homogenous as over $95 \%$ are members of the Luo tribe. Except for market places, the population in each village is highly dispersed, since the inhabitants live in family compounds surrounded by their fields. The main occupation of residents is in cultivation and limited animal husbandry made up of a few heads of cattle, goats or chicken. Activities off the farm include fishing in Lake Victoria and management of retail and hotel outlets and vegetables and grain stalls. There are two rainy seasons in this area: the long rains occur from March to May and the short rains from October to December. Malaria transmission is intense and occurs throughout the year [9]. The average number of infectious bites ranges between 60 and 300 per person per year. In a cohort of children monitored between 1992 to 1996, the < 5 year mortality rate was very high and estimated to be $25.9 \%$, with an infant mortality rate of 176/1000 [10]. While malnutrition is believed to be an important health problem, especially in the months before the harvest, estimates from this area have now been described [11,12].

\section{Cross-sectional surveys 1,2 and 3}

Between October 1999 and December 2003, three cross-sectional surveys were conducted as part of the bednet project to determine the impact of bednets on all cause 
morbidity in pre-school children [7]. The first cross-sectional survey represented the baseline survey and was conducted prior to the distribution of the bednets to intervention villages in December and during the short rains. The rainfall was the same as the average rainfall during that period in the previous 10 years (average daily rainfall in $\mathrm{mm}$ in the previous 3 months: $2.1 \mathrm{~mm}$ ). The second and third surveys were conducted in the months of February-March and November-December, 2004. All the three cross-sectional surveys were used to generate data reported here.

\section{Participant recruitment and study design}

The design of the baseline cross-sectional survey (survey-0) was different from the cross-sectional surveys after the start of the bednet intervention (survey-1 and survey2). Details of this are reported elsewhere [7, 12].

\section{Clinical Data on haemoglobin, parasites, malaria antibodies and malnutrition}

A finger-prick blood sample $(400 \mu \mathrm{l})$ was taken for determination of haemoglobin concentrations and the presence of malaria parasites. Sera from the blood was separated and used for assay of malaria antibodies using Enzyme Linked immunosorbent Assay (ELISA). Each child was examined by the clinical officer and the results recorded on standardised study forms. In survey-2, this included questions on the presence of abnormal hair and skin texture and bipedal pitting oedema. Periorbital and oedema of the hand were not assessed. All sick children were treated free of charge by the clinical officer. Severely malnourished children were referred to the local hospital and a nutritional feeding centre, which was centrally located in the study area.

\section{Anthropometric measurements (Height, Weight, Age and Mid upper-arm circumference)}

Anthropometric measurements were performed according to standard WHO procedure [13]. Children aged $<6$ months were undressed and weighed in plastic weighing pants to the nearest 10 grams using a $10 \mathrm{~kg} \pm 10 \mathrm{~g}$ hanging weighing scale (Salter, UK). Weight of the older children, wearing light clothes only was measured to the nearest 100 grams using a $25 \mathrm{~kg} \pm 100 \mathrm{~g}$ hanging weighing scale (CMS, UK). The weighing scales were calibrated daily. Recumbent length of children aged less than two years was measured to the nearest $0.1 \mathrm{~cm}$, using a horizontal measuring board with a sliding foot piece. Standing height was measured in the children from 2 years of age to the nearest $0.1 \mathrm{~cm}$ using a wooden length-measuring board with sliding head bar. Height and length were measured when children were barefoot and after removal of headgear [14]. Both height boards were manufactured by a local carpenter and were based on specifications of an internationally recommended design [13]. The Mid-upper Arm Circumference (MUAC) was measured to $0.1 \mathrm{~cm}$ using specialised non-stretchable measuring tapes (Zerfuss insertional tapes, Ross ltd, USA). All anthropometric measurements were taken by the same two members of the study team throughout each survey to minimize inter-observer variation. During survey 1 and survey 2 each measurement was taken twice and the mean computed. 


\section{Determination of Malaria Antibody Profiles}

Humoral immunity responses in plasmodium infection in relation to malnutrition status were studied. Enzyme linked immunosorbent assay was carried out to determine the plasmodium falciparum antibodies present in the study population by use of known antigens namely Liver Stage Antigen (LSA-1), Merozoite Surface Protein (MSP) and Circumsporozoite Surface Protein (CSP) [8] and to relate this to the protein energy malnutrition status [12]. Procedures in the assays were as previously described [14]. Definitions for the antigens were extensively covered elsewhere [7,8,12,15]. Liver Stage Antigen (LSA-1), Merozoite Surface Protein (MSP) and Circumsporozoite Surface Protein (CSP) were the preferred antigens for the assays.

\section{Data Management and Statistical Methods}

Data management was carried out as detailed in a different publication [7, 15]. Data were cleaned using range and internal consistency checks. Nutritional indices were generated with EPINUT (Epi Info v6, 2002 Atlanta, GA, USA). The analysis was weighted such that data from each survey contributed equally (33.3\% each) to the final data set in the < 36-month age groups. Only survey-0 and survey-2 contributed to the 36-59 month age group (50\% each). Differences in proportions were analysed using Chi-square tests or Fisher's exact test when appropriate, as well as the relative risk with 95\% confidence intervals. The kappa score was used to assess the level of agreement between the Cambridge and the WHO MUAC-for-age indices, as well as between the MUAC-for-age and the three other nutritional indices. Differences in proportions were analysed using the odds ratio (OR) with 95\% confidence intervals in the 3 cross- sectional surveys carried out at different times. As in survey 1 and survey 2 the randomisation was by household rather than by individual. Clustering of children at the household level was controlled for by age to obtain the $95 \%$ confidence intervals for the point estimates of the prevalence of the nutritional parameters as well as for the relative risks when comparing point estimates $[7,11,15]$. This was done using SUDAAN v8.0, SAS callable version, Research Triangle Institute, USA. Confidence intervals for rare outcomes $(<5 \%)$ were calculated using Exact methods (StatXact 4.0.1, Cytel, USA). All other analyses were conducted using SPSS (SPSS for Windows version 10.0.7 and 11.0, SPSS Inc). For all statistical tests, a two-sided P-value $<0.05$ was considered significant.

\section{Ethical Clearance and Informed Consent}

The bednet project was approved by the institutional review boards of the Kenya Medical Research Institute (KEMRI), Nairobi, Kenya and by the Centers for Disease Control and Prevention (CDC), Atlanta, USA. Written informed consent was obtained from caretakers for each individual participant.

\section{RESULTS}

Out of a total of 3575 children enrolled, only 2112 children were included in the final analysis. The remaining 1463 children did not satisfy the inclusion criteria or were not brought for assessment by their guardians. 


\section{Anthropometric indices}

These were extensively described in work already published elsewhere [6, 11, 12, 14]. Briefly, Z-score distributions for nutritional status as measured by the HAZ, WAZ as well as the WHZ were determined. Z-score values above the statistically defined cutoff of -2 SDs relative to the reference mean were then taken. The prevalence of any (<-2Z) and severe (<-3Z) stunting, wasting, underweight and low MUAC-for-age was associated to the malaria antibody profiles using correlation coefficient method. For MUAC : The two methods used to classify children as normal or lowMUAC-for-age, (based on the Cambridge and WHO reference, respectively), were in agreement (kappa $(\mathrm{k})=0.75)$.

\section{Anthropometry and malaria antibody profiles}

In CSF antibody profiles, higher percentages of IgG (44.4) and IgM (45.5) were observed in stunted children as compared to the wasted and underweight children. In LSA the IgG2, IgG3, IgG4 and IgM were also higher than in children who were wasted and those who were underweight. In MSP the IgG, IgG1 and IgG2 percentages in children who were stunted were almost similar to those in children who were wasted and underweight (Table 1).

\section{Association between PEM and malaria}

Univariate analysis showed the association between wasting and malaria parasitaemia to be not significant (Table 2b) $\mathrm{P}<0.001$, but was significant for stunting and underweight (Table 2a, b). Cross-tabulation of Stunting (HFA), Wasting (WFH) and underweight (WFA) with malaria antigens CSP, LSA and MSP showed stunting to be less significantly associated with all the antigens studied (Table 2b). The mean antibody concentrations and their controls showed CSP IgG antibody to be significantly associated with stunting $\mathrm{p}=0.022$, $(\mathrm{p}<0.05)$ but not with wasting. LSA IgG antibody was significantly associated with wasting only $\mathrm{p}=0.756,(\mathrm{p}<0.05)$ while MSP IgG antibody was not significantly associated with any malnutrition state $\mathrm{p}=0.0881$, $(\mathrm{p}<0.05)$. The mean concentration of CSP IgG was elevated in stunted, wasted and underweight children in comparison to controls. Liver Stage Antigen 1 IgG was elevated in stunted children only as compared to controls, whereas MSP IgG was low in all PEM cases as compared to controls.

\section{DISCUSSION}

This paper presents an assessment of malnutrition and its relationship with malaria antibodies as a possible diagnostic tool in a random sample of young children living in a rural area of western Kenya, on the shores of Lake Victoria. No such data from the study area were available to support this relationship though similar work has been reported from Senegal [16]. Further work published elsewhere explains the possible relationship between malnutrition and malaria from this area, but the data described relied heavily on the relationship between PEM and malaria morbidity and mortality in general without mentioning the role of antibodies in PEM in Western Kenya [7, 12]. 
This study confirms that PEM especially stunting (HAZ) may also be significantly associated with levels of specific malaria antibodies. These findings agree with the data from the study carried out in Senegal in 2009 [16].

According to the Senegal study, the prevalence of anti-malarial immune responders and specific IgG Ab levels were significantly associated with stunting. In this study, Circumsporozoite Surface Protein (CSP) IgG antibody was found to be significantly associated with stunting and underweight $(\mathrm{p}<0.05)$ but not with wasting, which agrees with the Senegal study [16]. This observation is further confirmed by work reported elsewhere using malaria parasitaemia indices instead of the antibody profiles [5]. Liver Stage Antigen (LSA) IgG antibody was significantly associated with wasting only $(\mathrm{p}<0.05)$ while MSP IgG antibody was not significantly associated with any malnutrition state, again confirming the data reported for malaria parasitaemia only [5]. The mean concentration of CSP IgG was elevated in stunted, wasted and underweight children in comparison to controls. Liverstage antigen 1 IgG was elevated in stunted children only as compared to controls, again confirming the observations made for the CSP antibody, whereas MSP IgG was low in all PEM cases as compared to controls, showing that malaria morbidity and mortality are affected by the PEM state. World Health Organization studies have also confirmed that children who were moderately to severely underweight had an increased but not statistically significant risk of a clinical malaria attack as compared to well nourished children [17]. Numerous other studies have demonstrated the role of specific IgG isotype responses in anti-malarial protective immunity. It is generally agreed that cytophilic IgG1 and IgG3 isotypes participate in specific protective immunity, whereas IgG2/IgG4 isotypes block its effect [16]. Malnutrition could regulate the balance between these protective/blocking isotype responses and consequently play a key role in the development of the protective anti-malarial immune response acquired during infection. This hypothesis is currently under investigation elsewhere [16].

In addition, nutritional status could modulate the immune response directed to malaria antigens, in particular, to major vaccine candidates. Indeed, a previous study showed that IgG Ab levels against RESA and Spf66 antigens were lower in wasted children compared to well-nourished children.

There were several constraints in the study. First, the study was conducted within the context of a large randomised controlled trial on permethrin treated bednets. As bednets are known to impact growth [18], positively all children living in villages using bednets were excluded from this analysis, which aimed to describe the prevalence of malnutrition in this community. Large scale use of bednets is recognised to exert a 'mass effect' or community effect [9,19,20,21]. Malaria morbidity was also reduced, specifically severe malarial anaemia and clinical malaria but there was no evidence for a mass effect on any of the standard nutritional parameters [21]. 


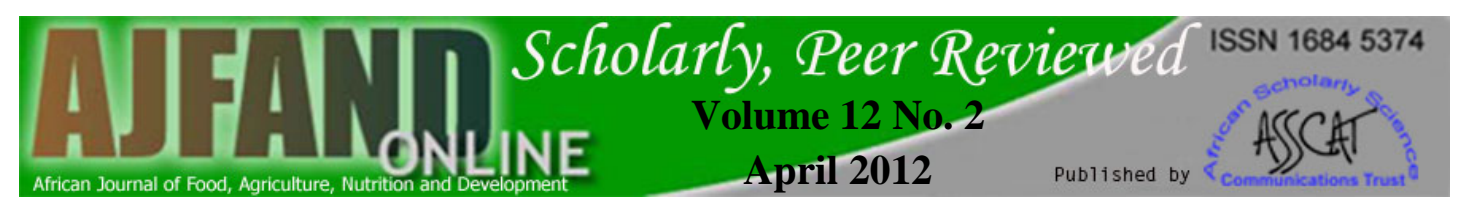

\section{CONCLUSION}

Interventions aimed at reducing child malnutrition in populations like the one reported in this paper should attempt to include all children $<2$ years of age in the community to elucidate clearly the role of malaria antibodies in PEM. Specific Plasmodium falciparum Antibody profiles could accurately be used to determine their diagnostic potential as well as understanding their potential role in the immunity of children with PEM. Therefore, future vaccine trials including major candidates should take into account child nutritional status when evaluating the specific immune response acquired after immunization

\section{ACKNOWLEDGEMENT}

We express our gratitude to the parents and guardians of the children who participated in the study and the many people that assisted with this project. Dr. Arthur Kwena acknowledges support from the Netherlands Organisation for Co-operation in Higher education (NUFFIC), the Netherlands. We thank the Director of Kenya Medical Research Institute (KEMRI) for permission to publish this work. 


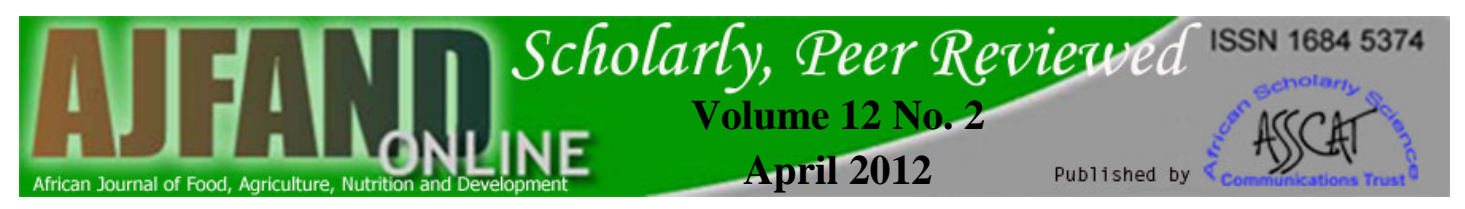

Table 1: Prevalence of Positive Titres to Schizont extract and Plasmodium Falciparum Proteins MSP 1, LSA 1, and CSP 1, by nutritional status in Asembo, Western Kenya

\begin{tabular}{|c|c|c|c|c|c|c|}
\hline & \multicolumn{2}{|c|}{ Stunted $(<-2$ ZHFA) } & \multicolumn{2}{|c|}{ Wasted (<-2ZWFH) } & \multicolumn{2}{|c|}{$\begin{array}{l}\text { Underweight } \\
\text { (<-2ZWFA) }\end{array}$} \\
\hline & $\mathrm{N}$ & $\%$ & $\mathrm{~N}$ & $\%$ & $\mathrm{~N}$ & $\%$ \\
\hline CSF IgG & 212 & 44.4 & 30 & 31.3 & 169 & 42.8 \\
\hline IgM & 231 & 48.3 & 33 & 34.4 & 170 & 42.8 \\
\hline LSA1IgG1 & 105 & 21.1 & 20 & 19.8 & 79 & 19.2 \\
\hline IgG2 & 33 & 6.6 & 5 & 5 & 28 & 6.8 \\
\hline IgG3 & 150 & 30.2 & 18 & 17.8 & 105 & 25.5 \\
\hline IgG4 & 37 & 7.4 & 5 & 5 & 22 & 5.3 \\
\hline IgM & 173 & 34.8 & 24 & 23.9 & 126 & 30.6 \\
\hline MSP1IgG & 108 & 22.9 & 18 & 18.9 & 83 & 21 \\
\hline IgG1 & 94 & 19.9 & 17 & 17.9 & 77 & 19.5 \\
\hline IgG2 & 20 & 4.2 & 5 & 5.3 & 12 & 3 \\
\hline Igg3 & 101 & 21.4 & 17 & \begin{tabular}{|l|}
17.9 \\
\end{tabular} & 95 & 24.1 \\
\hline IgG4 & 44 & 9.3 & 7 & 7.4 & 35 & 8.9 \\
\hline
\end{tabular}

Higher percentages of IgG and IgM were observed in stunted children as compared to the wasted and underweight children

IgG2, IgG3, IgG4 and IgM were also higher than in children who were wasted and those who were underweight

IgG, IgG1 and IgG2 percentages in children who were stunted were almost similar to those in children who were wasted and underweight 


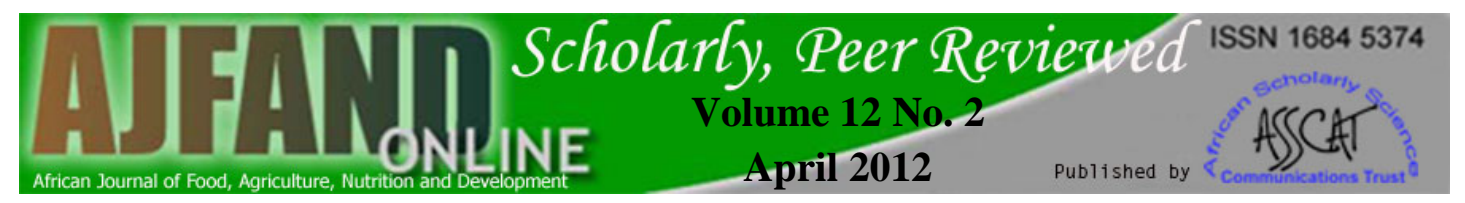

Table 2: Interaction between 2a) Stunting and Malaria 2b) Wasting and Malaria 2c) Underweight and Malaria

2a) Stunting and Malaria

\begin{tabular}{|l|c|c|c|}
\hline Variable category & N & OR (95\%CI) & p-value \\
\hline Age & & - & $<0.001$ \\
\hline $0-5$ & 25 & - & - \\
\hline $6-23$ & 359 & - & - \\
\hline \multicolumn{1}{|c|}{$>$ YY } & 576 & - & - \\
\hline Sex & & & 0.010 \\
\hline T37.5 & 502 & $1.2(1.0,1.3)$ & - \\
\hline Para + palpable spleen & 72 & - & $<0.001$ \\
\hline Positive malaria smear & 256 & $(0.7,1.5)$ & $<0.001$ \\
\hline Parclin & 680 & - & 0.449 \\
\hline Fevclin & 253 & $(0.8,1.6)$ & - \\
\hline \multicolumn{1}{|c|}{ 5000/mm3 } & 37 & - & $<0.001$ \\
\hline CSP IgG tot pos/neg & 257 & $(1.0,1.8)$ & $<0.001$ \\
\hline LSA IgG tot pos/neg & 212 & - & 0.981 \\
\hline MSP 1 tot pos/neg & 135 & - & 0.087 \\
\hline
\end{tabular}

$\mathrm{P}<0.001$ was considered significant

2b) Wasting and Malaria

\begin{tabular}{|c|c|c|c|}
\hline Variable category & $\mathbf{N}$ & OR (95\%CI) & p-value \\
\hline Age & & - & 0.021 \\
\hline $0-5$ & 11 & - & - \\
\hline $6-23$ & 103 & - & - \\
\hline$>=2 Y$ & 61 & - & - \\
\hline Sex & 107 & $1.7(1.3,2.2)$ & 0.001 \\
\hline \multicolumn{4}{|l|}{$\mathrm{M}$} \\
\hline T37.5 & 19 & $(1.3,3.0)$ & 0.002 \\
\hline Para + palpable spleen & 33 & $=$ & 0.881 \\
\hline Positive malaria smear & 99 & - & 0.334 \\
\hline Parclin & 37 & $(0.8,1.6)$ & 0.815 \\
\hline Fevclin & 7 & - & 0.541 \\
\hline$>5000 / \mathrm{mm} 3$ & 42 & $(1.0,1.8)$ & 0.314 \\
\hline CSP IgG tot pos/neg & 30 & - & 0.220 \\
\hline LSA IgG tot pos/neg & 29 & $=$ & 0.756 \\
\hline MSP 1 tot pos/neg & 18 & - & 0.881 \\
\hline
\end{tabular}

$\mathrm{P}<0.001$ was considered significant. 


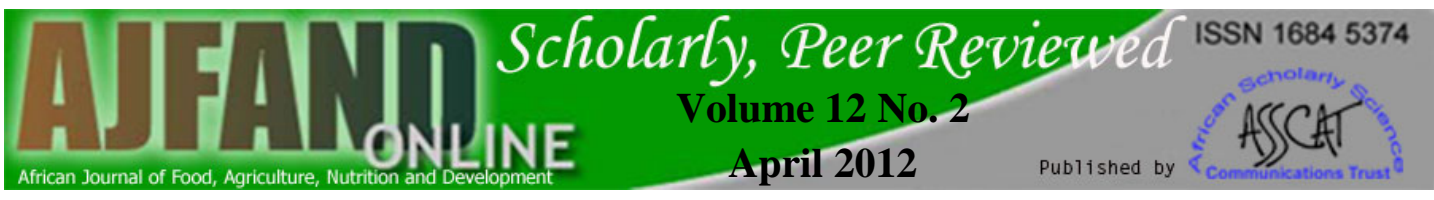

2c) Underweight and Malaria

\begin{tabular}{|c|c|c|c|}
\hline Variable category & $\mathbf{N}$ & OR (95\% CI) & p-value \\
\hline \multicolumn{4}{|l|}{ Age } \\
\hline $0-5$ & 36 & - & $<0.001$ \\
\hline $6-23$ & 359 & - & - \\
\hline$>=2 Y$ & 400 & - & - \\
\hline Sex & 412 & $1.1(1.0,1.3)$ & $0.020 *$ \\
\hline $\mathrm{M}$ & - & - & - \\
\hline T37.5 & 77 & $(1.3,2.2)$ & $<0.001$ \\
\hline Para + palpable spleen & 191 & $(1.1,1.6)$ & 0.003 \\
\hline Positive malaria smear & 543 & - & $<0.001$ \\
\hline Parclin & 211 & $(1.3,1.8)$ & $<0.001$ \\
\hline Fevclin & 39 & - & 0.002 \\
\hline$>5000 / \mathrm{mm} 3$ & 229 & $(1.5,2.1)$ & $<0.001$ \\
\hline CSP IgG tot pos/neg & 169 & - & 0.010 \\
\hline LSA IgG tot pos/neg & 107 & - & 0.502 \\
\hline MSP 1 tot pos/neg & 83 & - & 0.017 \\
\hline
\end{tabular}

Parasitaemia (Pos/Neg)- malaria smear computed as positive or negative

Parclin- Parasitaemia/ $/ \mathrm{mm}^{3}$ above fever threshold

Fevclin- Fever + parasitaemia $>$ fever threshold

$>5000 / \mathrm{mm}^{3}$-Parasitaemia cut-off of $5000 \mathrm{~mm}^{3}$ 


\section{REFERENCES}

1. Bern C, Zucker JR, Perkins BA, Otieno J, oloo AJ and R Yip assessment of potential indicators for protein-energy malnutrition in the algorithm for integrated management of childhood illness. Bull. WHO 1997; 75: 87-96.

2. Bloland PB, Lackritz EM, Kazembe PN, Were JBO, Steketee R and CC Campbell Beyond chloroquine: implications of drug resistance for evaluating malaria therapy, efficacy and treatment policy in Africa. J. Infect. Dis. 1993; 167: 932-937.

3. Bradley-Moore AM, Greenwood BM and AK Bradley Malaria chemoprophylaxis with chloroquine in young Nigerian children 111: its effect on nutrition. Ann. Trop. Med. Parasitol. 1985; 79: 575-584.

4. Dominguez-Vazquez A and A Alzate-Sanchez Nutritional status in children under 6 years of age and its relation to malaria and intestinal parasitism. Salud Publica Mex. 1990; 32: 52-63.

5. Friedman JF, Kwena A, Mirel LB, Kariuki SK, Terlouw DJ, PhillipsHoward PA, Hawley WA, Nahlen BL, Ya Ping Shi and FO Ter Kuile Risk factors for Protein-Energy Malnutrition among young children in an area of intense perennial malaria transmission in Western Kenya: Results of cross sectional survey. Am. J. Trop. Med. Hyg. 2005; 73(4): 698-704.

6. Theresa K, Nkuo- Akenji, Sumbele I, Mankah EN, Njunda AL, Samje M and L Kamga The Burden of Malaria and Malnutrition among children less than 14 years of age in a rural village of Cameroon. African Journal of Food Agriculture Nutrition and Development 2008; 8 (3): 252-264.

7. Ter Kuile FO, Terlouw DJ, Phillips-Howard PA, Hawley WA, Friedman JF, Kariuki S, Ya Ping Shi, Kwena A, Vulule $\mathbf{J}$ and BL Nahlen Impact of Permethrin treated bednets on malaria and all cause morbidity in young children in an area of intense perennial malaria transmission in western Kenya: crosssectional survey. Am. J. Trop. Med. Hyg. 2003; 68 (4): 100-107.

8. Kariuki SK, Lal AA, Terlow DJ, Ter Kuile FO, Ong'echa JMO, PhillipsHoward PA, Orago SSA, Kolczak MS, Hawley WA, Nahlen BL and YP Shi Effects of permethrin treated bednets on malaria immunity in western Kenya ii: Antibody responses in young children in an area of intense malaria transmission. Am. J. Trop. Med. Hyg. 2003; 68: 08-114.

9. Phillips-Howard PA,Nahlen BL, Kolczak MS, Hightower AW, Ter Kuile FO, Alaii JA, Gimnig JE Arudo J, Vulule JM, Odhacha A, Kachur PS, Schoute E, Rosen DH, Sexton JD, Oloo AJ and WA Hawley Efficacy of permethrin- treated bed nets in the prevention of mortality in young children in an area of high perennial malaria transmission in western Kenya. Am. J. Trop. Med. Hyg. 2003; 68:23-29. 
10.. Bloland PB, Ruebush TK, McCormick JB, Ayisi J, Boriga DA and AJ Oloo Longitudinal cohort study of the epidemiology of malaria infections in an area of intense malaria transmission 1. Description of study site, general methodology and study population. Am. J. Trop. Med. Hyg. 1999; 60: 635-640.

11. McElroy PD, Ter Kuile FO, GHightower AW, Hawley WA, PhillipsHoward PA and AJ Oloo All-cause mortality among young children in western Kenya, VII: The Asembo Bay Cohort Project. Am. J. Trop. Med. Hyg. 2001; 64: 18-27.

12. Kwena A, Terlouw DJ, de Vlas SJ, Phillips-Howard PA, Hawley WA, Friedman JF, Vulule J, Nahlen BL, Sauerwein RW and FO ter Kuile Prevalence and severity of malnutrition in pre-school children in a rural area in Western Kenya. Am. J. Trop. Med. Hyg. 2003; 68:(4): 94-99.

13. United Nations. Summary procedures how to weigh and measure children, In: Assessing the nutritional status of young children in household surveys. (ed.) United Nations, New York, 1986, p 1-11.

14. Hautvast JLA, Tolboom JJM, Kafwembe EM, Musonda RM, Mwanakasale V, Staveren WA, van't Haf MA, Sauerwein RW, Willems JL and LAH Monnens Severe linear growth retardation in Zambian children: the influence of biological variables. Am. J. Clin. Nutr. 2000; 71: 550-559.

15. Desai MR, Terlouw DJ, Kwena AM, Phillips-Howard PA, Kariuki SK, Wannemuehler KA, Odhacha A, Hawley WA, Ya Ping Shi, Nahlen BL and FO ter Kuile Factors associated with hemoglobin concentrations in pre-school children, western Kenya: cross-sectional studies. Am. J. Trop. Med. Hyg. 2005; 72 (1): 47-59.

16. Fillol F, Sarr JB, Baulanger D, Cisse B, Sokhna C, Riveau G, Simondon KB and $\mathbf{F}$ Remou Impact of child malnutrition on the specific anti-Plasmodium falciparum antibody response. Malaria Journal 2009: 8: 116-135.

17. Caulfield LE, Richard SA and RE Black Undernutrition as an underlying cause of malaria morbidity and mortality in children less than five years old. Am J Trop Med Hyg 2004; 71: 55-63.

18. Snow RW, Molyneux CS, Njeru EK, Omumbo J, Nevill CG and E Muniu The effects of malaria control on nutritional status in infancy. Acta Tropica 1997; 65:1-10

19. Binka FN, Indome $\mathbf{F}$ and $\mathbf{T}$ Smith Impact of spatial distribution of permethrin-impregnated bed nets on child mortality in rural Northern Ghana. Am. J. Trop. Med. Hyg. 1988; 59: 80-85. 


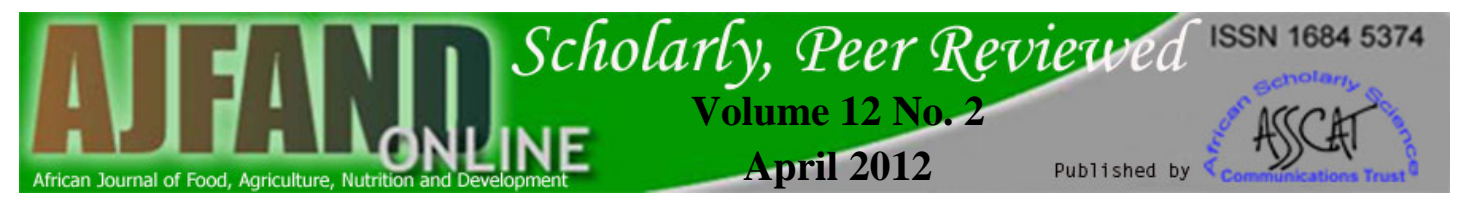

20. Howard SC, Omumbo J, Nevill C, Some ES, Donelly CA and RW Snow Evidence for mass community effect of insecticide treated bed nets on incidence of malaria on Kenya coast. Trans. Roy. Soc. Trop. Med. Hyg. 2000; 94: 357360 .

21.. Hawley WA, Phillis-Howard PA, Ter Kuile FO, Terlow DJ, Vulule JM, Ombok M, Nahlen BL, Gimnig JE, Kariuki SK and MS Kolczak Hightower AW, Community wide effects of permethrin treated bed nets on child mortality and malaria morbidity in western Kenya. Am. J. Trop. Med. Hyg. 2003; 68: 121-127. 